\title{
Geometric optimization of steel trusses with parallel chords
}

\author{
M. Kripka*1 , G.A. Drehmer ${ }^{2}$ \\ ${ }^{1}$ University of Passo Fundo, Civil and Environmental Engineering Graduate Program, Passo Fundo, \\ Brazil \\ ${ }^{2}$ Director of Grid Structural Engineering, Passo Fundo, Brazil
}

\begin{abstract}
The application of optimization to the solution of practical structural engineering problems has been very limited, despite the great development of the techniques. One of the main reasons for this is the complexity of the generated models, which employ nonlinear functions and generate a space of nonconvex solutions. In addition, most design variables, as in the case of steel profiles, can only assume discrete values. Traditional methods of mathematical programming are very limited in solving problems with these characteristics, opening space to the usage of heuristic methods and, more specifically, to metaheuristic methods. The main advantage of this class of methods is the fact that they only involve values of the functions in the optimization process, regardless of the existence of unimodality or even continuity in the derivatives of the functions involved. The present work presents the application of a heuristic method, the simulated annealing method, to the optimization of steel trusses with parallel chords, also called flat trusses. Initially, several usual configurations of trusses were analyzed, aiming to identify which led to the lowest cost. The top chord coordinates were also included as design variables, with the purpose of verifying the relations between span and height normally indicated in the literature as the most economical. Among the results, it was verified that the inclusion of the height of the truss among the design variables can lead to a significant additional cost savings.
\end{abstract}

\section{Keywords}

Truss; Optimization; Parallel chords; Weight; Steel; Geometry

Received: 21 September 2018; Accepted: 02 October 2018

ISSN: 2630-5771 (online) C 2018 Golden Light Publishing All rights reserved.

\section{Introduction}

The analysis and design of structures are essentially based on iterative procedures. Because of this, the values initially adopted for the design variables depend fundamentally on the sensitivity and the previous experience of the calculator. However, when working with a large number of variables, the number of combinations increases exponentially when it is desired to obtain the values that result in the best design among all alternatives. However, when the physical problem is described through mathematical functions, optimization techniques can be used to search for the extreme values of these functions.

The main reason usually posited to explain the sparse application of optimization techniques to practical structural engineering problems is the complexity of the generated models, which employ nonlinear functions and frequently generate a space of nonconvex solutions (several points of minimum). Because mathematical programming techniques have proved to be inefficient in solving

\footnotetext{
Corresponding author

Email: mkripka@upf.br
} 
these kinds of problems, it is common to introduce some simplifications in the formulation to simplify the problem. In the specific case of truss optimization, the focus of the present work, common simplifications found in the technical literature include the treatment of the cross sections of the elements as continuous variables, the disregard of buckling, non-inclusion of several loading cases, as well as their combinations, and the absence of limits on displacements or the verification of the elements based on allowable stresses. It must be emphasized, however, that the introduction of one or more of these simplifications may lead to unrealistic solutions, which will require a series of additional checks by the user, making it difficult or even impossible for practical application of the obtained results. With the aim of solving more complex problems, heuristic methods are playing an increasingly important role, because they only involve the values of the functions in the analysis, without the requirement that they present certain characteristics such as unimodality or even continuity of their derivatives. However, heuristic methods have as disadvantages a large number of function evaluations and the lack of assurance for most of these methods that the solution effectively converges to an optimal point. A huge number of heuristic methods have been proposed in the past few decades, but most of them are just a slight variation of previous methods [1,2]. Among the main consolidated heuristic methods is the simulated annealing method, which consists of a global search procedure developed in analogy to the metal annealing process and originally proposed by Kirkpatrick et al. [3]. Some advantages of the simulated annealing method are its easy computational implementation and the reduced number of control parameters compared, e.g., to genetic algorithms, another important and widely used optimization method.

This work presents the minimization of steel trusses of parallel chords (also called flat trusses), composed of rolled profiles. For this purpose, the cross sections of the elements are treated as discrete variables and dimensioned according to the Brazilian standard [4]. First, some usual configurations are analyzed in an effort to identify which configuration leads to the lowest total cost for a given load. The coordinates of the upper chord are also included as design variables, with their influence in the reduction of the cost of the structure as well as the economic relations between the height and the span of the truss being studied.

Several studies have been developed recently regarding truss optimization. Most of them address the development and/or improvement of optimization techniques, and an extensive review is presented by Stolpe [5]. However, most of these studies consider just benchmark trusses, without promoting any improvement in real-life structures. In our work, besides the application of the optimization technique, we also aim to generate some relations to be used by engineers in their current practice. Although considering just a specific steel profile (L-shaped with a double angle of equal and opposite legs), we suggest that the conclusions obtained can be extended to other profiles. In addition, because cost and weight are closely related to steel structures, natural variations in the unit price of steel does not alter significantly the results.

This remainder of the paper is structured as follows. Section 2 presents the formulation of the optimization problem. Section 3 presents the structures analyzed and the corresponding results. The conclusions and general considerations are presented in Section 4.

\section{Formulation of the optimization problem}

The objective of the developed formulation is to determine the cross sections of the elements and the nodal coordinates that lead to the lowest $\operatorname{cost} C$ for the structure, while addressing the limit states (ultimate and serviceability). The corresponding objective function $f(x)$ is described for a structure composed of $n$ elements as

$\operatorname{minimize} f(x)=C=U c \cdot P$

where $U c$ is the unit cost $(\$ / \mathrm{kg}$, where all dollars quoted herein are in US dollars) and $P$ is the total weight of the structure. The weight is obtained as a sum of the volume of each element multiplied by its 
specific weight (here considered constant and equal to $77 \mathrm{kN} / \mathrm{m}^{3}$ ).

Because of the practical adoption of the same profile for a group of elements, the number of design variables is drastically reduced in relation to the total number of elements. For the simultaneous optimization of geometry and sections, the set of design variables comprises the sum of the number of groups of elements and the number of groups of displaceable nodes. In the case of the proposed strategy, the values for the available cross sections of a group of elements are chosen from a table of profiles previously defined, composing therefore a set of discrete variables. Nodal coordinates are also treated as discrete and could assume multiple values of $1 \mathrm{~cm}$, within a limited range.

The problem addressed is subject to the following constraints, expressed in normalized form as

$g_{1}=\frac{s_{d}}{R_{d}}-1 \leq 0$

$g_{2}=\frac{\lambda}{\lambda_{\text {lim }}}-1 \leq 0$

$g_{3}=\frac{\delta}{\delta_{\text {lim }}}-1 \leq 0$

where $S_{d}$ is the maximum internal force in each group of elements, $R_{d}$ is the maximum cross-section strength of each group, considering local and global buckling for compressed elements, $\lambda$ is the slenderness of the element, and $\lambda_{\text {lim }}$ is the allowable slenderness (with different values for tension and compression assumed). Eq. (4) is related to the serviceability limit state, with $\delta$ being the displacements in each node and $\delta_{\text {lim }}$ their corresponding limits. In cases of more than one load combination, the total number of problem constraints is multiplied by the number of combinations.

The proposed formulation was implemented by combing the simulated annealing method with the displacement method for the analysis of truss structures. The consideration of the constraints in the computational implementation was made by using a dynamic penalty factor, called an annealing penalty [6]. As in the simulated annealing method, the penalty factor has a high initial temperature, and therefore the penalty is relatively low. As the temperature is reduced, smaller violations are allowed, and at the end of the process only feasible solutions (that meet the constraints) are accepted. The penalty function $F(x)$ is written as

$F(x)=f(x)+\phi(x)$

where

$\phi(x)=\sum\left(\frac{1}{2 \mathrm{~T}}\right) \mathrm{g}(\mathrm{x})^{2}$

In the previous expression, $\phi(x)$ is the penalty function of the violated constraints for the current solution. According to this, even when the problem starts from a nonfeasible solution, small violations of the restrictions are accepted. In this way, the problem becomes less dependent on the initial solution (initial values assigned to the cross sections and nodal coordinates of the displaceable nodes). The same strategy was adopted successfully by the authors in previous works [7-10].

\section{Parallel chord truss optimization}

Despite the generality of the developed formulation, the examples presented were developed to study the optimum configuration and geometry of parallel chord trusses (flat trusses). Initially, we tried to identify, among some usual configurations, the one that led to the lowest total cost. Next, we also included the coordinates of the upper chord nodes as design variables. Finally, for one of the configurations, the relation obtained between the height and the length of the span is presented to endorse the relations usually suggested as the most economical.

All structures that we analyzed have several characteristics in common. The studies started from plain trusses with a total span of $9 \mathrm{~m}$ and a distance between chords of $1 \mathrm{~m}$. The loads were applied at the upper nodes, which were spaced $1.50 \mathrm{~m}$ apart (Fig. 1). 


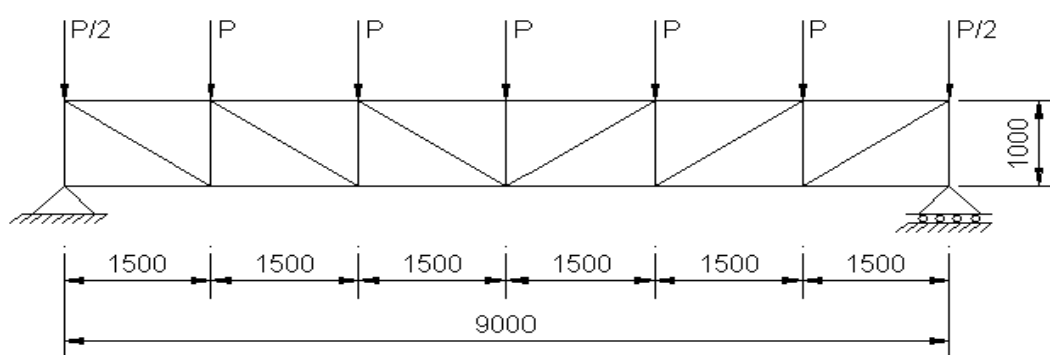

Fig. 1. Geometry of the trusses (with dimensions in millimeters)

For the composition of the load, we considered the actions applied at the nodes with the following values: dead load $G=1.2 \mathrm{kN}$, live load $Q 1=2.0$ $\mathrm{kN}$, and wind $Q 2=-7.5 \mathrm{kN}$. The combinations considered are the following:

Combination $\mathrm{C} 1: 1.3 G+1.5 Q 1 \Rightarrow P=4.56 \mathrm{kN}$ and

Combination $\mathrm{C} 2$ : $0.9 G+1.4 Q 2 \Rightarrow P=-9.42 \mathrm{kN}$.

The elements were dimensioned with rolled profiles in the form of an $\mathrm{L}$ shape with a double angle of equal and opposite legs. These profiles, listed in Table 1, constitute a total of 33 sections. The characteristics to be supplied to the program are also listed in Table 1 . They are height $b$, thickness $t$, cross-sectional area of the composite profile, $A$, and moment of inertia with respect to principal axis, $I_{x}$. It should be pointed that the outof-plane bracing at all nodes was considered. In this way, only the properties with respect to the main horizontal $x$ axis (Fig. 2) are necessary, regardless of the distance $a$ between profiles.

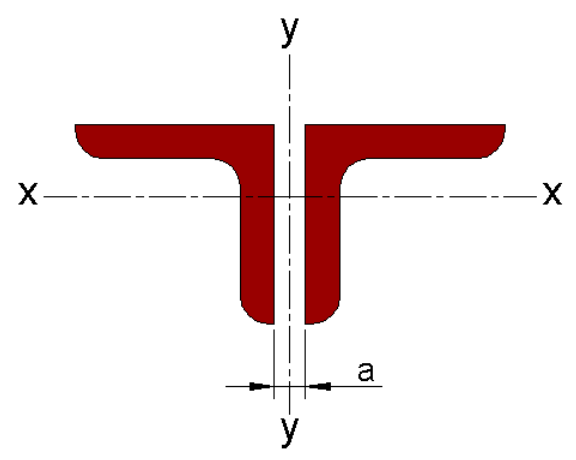

Fig. 2. Profiles adopted in the analyses

\subsection{Cross section optimization}

Using the described geometry and loading conditions, the proposed procedure for the optimization of the cross sections of trusses was applied according to the configurations indicated in Fig. 3.

First, we sought to identify, among the studied configurations, the one that led to the lowest cost, considering the same cross section for all elements of a structure (i.e., a single group of elements). The results obtained are summarized in Table 2. Here $A^{*}$ is the optimum cross section for each configuration, according to the profile number (given in the first column of Table 1) with its identification in parentheses, $P^{*}$ is the total weight corresponding to the profile, and $C^{*}$ is the total cost. Also in Table 2, the total number of elements, $n$, is indicated, as well as where the maximum stress occurred (UC indicating an upper chord element and LC a lower chord element).

For all the analyzed situations, the internal forces resulting from the second loading combination were the determining factors for the dimensioning of the elements. It was observed that, despite the greater number of elements, configuration $\mathrm{E}$ generated the lowest cost, owing to the shorter length of the elements of the lower chord (compressed elements). For all other configurations, because the optimal cross section was the same, the determinant factor for the greater or lesser cost was only the total length of the elements.

Similar analyses were performed for the same structures by varying the number of groups of elements from one to four groups. The corresponding costs are given in Table 3 as follows: 
$C_{1} *$ : the same section to all elements (as considered above);

$C_{2} *$ : the same section to the chords and others to the remaining elements (diagonals and verticals);

Table 1. Characteristics of adopted profiles

\begin{tabular}{|c|c|c|c|c|c|}
\hline Profile number & Identification (pol) & $b(\mathrm{~mm})$ & $t(\mathrm{~mm})$ & $A\left(\mathrm{~cm}^{2}\right)$ & $I_{x}\left(\mathrm{~cm}^{4}\right)$ \\
\hline 1 & $2 \mathrm{~L}^{5} / 8^{\prime \prime} \times 1 / 8^{\prime \prime}$ & 16 & 3.2 & 1.92 & 0.40 \\
\hline 2 & $2 \mathrm{~L} 3 / 4^{\prime \prime} \times 1 / 8^{\prime \prime}$ & 19 & 3.2 & 2.32 & 0.74 \\
\hline 3 & $2 L^{7} / 8^{\prime \prime} \times 1 / 8^{\prime \prime}$ & 22 & 3.2 & 2.70 & 1.16 \\
\hline 4 & $2 \mathrm{~L} 1^{\prime \prime} \times 1 / 8^{\prime \prime}$ & 25 & 3.2 & 2.96 & 1.66 \\
\hline 5 & $2 \mathrm{~L} 11^{1 / 4^{\prime \prime}} \times 1 / 8^{\prime \prime}$ & 32 & 3.2 & 3.86 & 3.32 \\
\hline 6 & $2 \mathrm{~L} 1^{\prime \prime} \times 3 / 16^{\prime \prime}$ & 25 & 4.8 & 4.38 & 2.48 \\
\hline 7 & $2 \mathrm{~L} 1 \frac{1}{2^{\prime \prime}} \times 1 / 8^{\prime \prime}$ & 38 & 3.2 & 4.64 & 6.60 \\
\hline 8 & $2 \mathrm{~L} 13 / 4^{\prime \prime} \times 1 / 8^{\prime \prime}$ & 44 & 3.2 & 5.40 & 10.82 \\
\hline 9 & $2 \mathrm{~L} 11^{1 / 4^{\prime \prime}} \times 3 / 16^{\prime \prime}$ & 32 & 4.8 & 5.54 & 4.98 \\
\hline 10 & $2 \mathrm{~L} 1^{\prime \prime} \times 1 / 4^{\prime \prime}$ & 25 & 6.3 & 5.66 & 3.32 \\
\hline 11 & $2 \mathrm{~L} 2^{\prime \prime} \times 1 / 8^{\prime \prime}$ & 51 & 3.2 & 6.18 & 15.80 \\
\hline 12 & $2 \mathrm{~L} 1 \frac{1}{2} 2^{\prime \prime} \times 3 / 16^{\prime \prime}$ & 38 & 4.8 & 6.84 & 9.14 \\
\hline 13 & $2 \mathrm{~L} 11^{1 / 4^{\prime \prime}} \times 1 / 4^{\prime \prime}$ & 32 & 6.3 & 7.22 & 6.64 \\
\hline 14 & $2 \mathrm{~L} 13 / 4^{\prime \prime} \times 3 / 16^{\prime \prime}$ & 44 & 4.8 & 7.98 & 14.98 \\
\hline 15 & $2 \mathrm{~L} 1 \frac{1 / 2^{\prime \prime} \times 1 / 4^{\prime \prime}}{}$ & 38 & 6.3 & 8.90 & 11.64 \\
\hline 16 & $2 \mathrm{~L} 2^{\prime \prime} \times 3 / 16^{\prime \prime}$ & 51 & 4.8 & 9.16 & 22.46 \\
\hline 17 & $2 \mathrm{~L} 13 / 4^{\prime \prime} \times 1 / 4^{\prime \prime}$ & 44 & 6.3 & 10.44 & 19.14 \\
\hline 18 & $2 \mathrm{~L} 2 \frac{1 / 2 "}{2^{\prime \prime}} \times 3 / 16^{\prime \prime}$ & 63 & 4.8 & 11.60 & 46.00 \\
\hline 19 & $2 \mathrm{~L} 2^{\prime \prime} \times 1 / 4^{\prime \prime}$ & 51 & 6.3 & 12.12 & 29.12 \\
\hline 20 & $2 \mathrm{~L} 3^{\prime \prime} \times 3 / 16^{\prime \prime}$ & 76 & 4.8 & 14.06 & 80.00 \\
\hline 21 & $2 \mathrm{~L} 2^{\prime \prime} \times 5 / 16^{\prime \prime}$ & 51 & 8.0 & 14.82 & 34.96 \\
\hline 22 & $2 \mathrm{~L} 2^{1} \frac{1}{2}{ }^{\prime \prime} \times 1 / 4^{\prime \prime}$ & 63 & 6.3 & 15.36 & 58.20 \\
\hline 23 & $2 \mathrm{~L} 3^{\prime \prime} \times 1 / 4^{\prime \prime}$ & 76 & 6.3 & 18.58 & 100.00 \\
\hline 24 & $2 \mathrm{~L} 2 \frac{1 / 2{ }^{\prime \prime}}{} \times 5 / 16^{\prime \prime}$ & 63 & 8.0 & 18.96 & 70.80 \\
\hline 25 & $2 \mathrm{~L} 2^{\prime \prime} \times 3 / 8^{\prime \prime}$ & 51 & 9.5 & 22.32 & 81.60 \\
\hline 26 & $2 \mathrm{~L} 2 \frac{1}{2^{\prime \prime}} \times 3 / 8^{\prime \prime}$ & 63 & 9.5 & 22.32 & 81.60 \\
\hline 27 & $2 \mathrm{~L} 3^{\prime \prime} \times 5 / 16^{\prime \prime}$ & 76 & 8.0 & 22.96 & 124.00 \\
\hline 28 & $2 \mathrm{~L} 4^{\prime \prime} \times 1 / 4^{\prime \prime}$ & 102 & 6.3 & 25.02 & 250.00 \\
\hline 29 & $2 \mathrm{~L} 3^{\prime \prime} \times 3 / 8^{\prime \prime}$ & 76 & 9.5 & 27.22 & 150.00 \\
\hline 30 & $2 \mathrm{~L} 4^{\prime \prime} \times 5 / 16^{\prime \prime}$ & 102 & 8.0 & 30.96 & 308.00 \\
\hline 31 & $2 \mathrm{~L} 3^{\prime \prime} \times 1 / 2{ }^{\prime \prime}$ & 76 & 12.7 & 35.48 & 182.00 \\
\hline 32 & $2 \mathrm{~L} 4^{\prime \prime} \times 3 / 8^{\prime \prime}$ & 102 & 9.5 & 37.14 & 366.00 \\
\hline 33 & $2 \mathrm{~L} 4^{\prime \prime} \times \frac{1}{2}{ }^{\prime \prime}$ & 102 & 12.7 & 48.38 & 466.00 \\
\hline
\end{tabular}

$C_{3} *$ : a section to the upper chord, a section to the lower chord, and a section to the remaining elements (diagonals and verticals);

$C_{4} *$ : a section to the upper chord, a section to the lower chord, a section to the diagonals, and a section to vertical elements. 


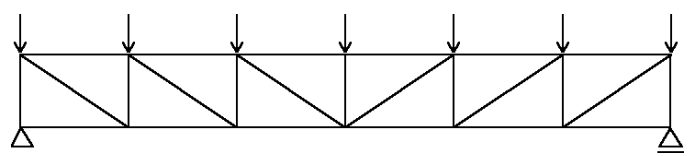

a) Configuration $\mathrm{A}$

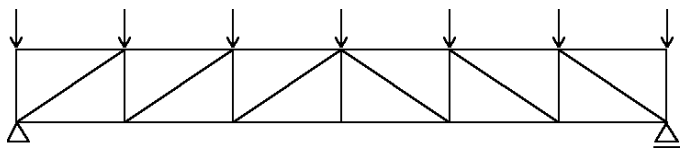

b) Configuration $\mathrm{B}$

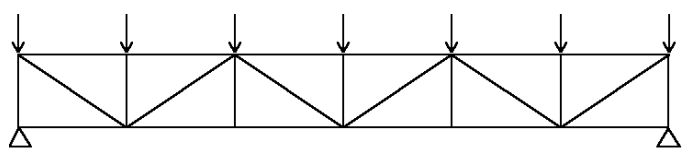

d) Configuration $\mathrm{D}$

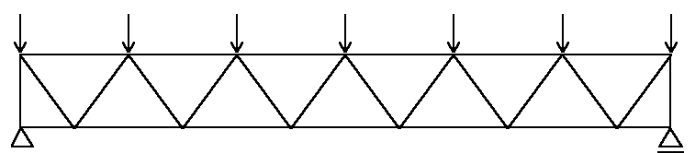

f) Configuration $F$

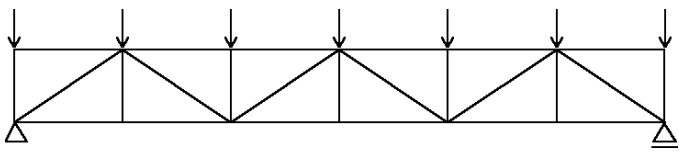

c) Configuration $\mathrm{C}$

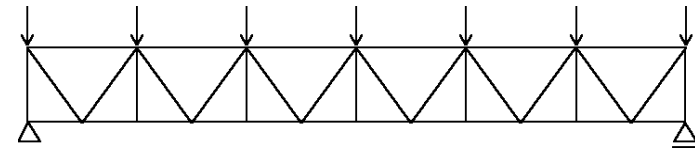

e) Configuration $\mathrm{E}$

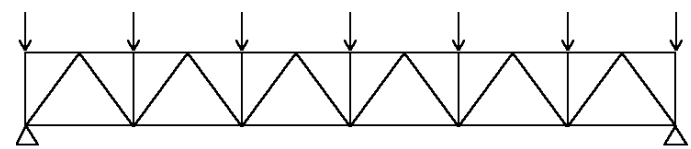

g) Configuration $\mathrm{G}$

Fig. 3. Configurations analyzed

Table 2. Results of a unique group of elements

\begin{tabular}{cccccc}
\hline Configuration & $A^{*}$ and corresponding profile & $P^{*}(\mathrm{kN})$ & $C^{*}(\$)$ & $n$ & Maximum stress \\
\hline A & $11\left(2 \mathrm{~L} 2^{\prime \prime} \times 1 / 8^{\prime \prime}\right)$ & 1.7044 & 490.87 & 25 & UC \\
B & $11\left(2 \mathrm{~L} 2^{\prime \prime} \times 1 / 8^{\prime \prime}\right)$ & 1.7044 & 490.87 & 25 & LC \\
C & $11\left(2 \mathrm{~L} 2^{\prime \prime} \times 1 / 8^{\prime \prime}\right)$ & 1.7044 & 490.87 & 25 & LC \\
D & $11\left(2 \mathrm{~L} 2^{\prime \prime} \times 1 / 8^{\prime \prime}\right)$ & 1.7044 & 490.87 & 25 & UC \\
E & $7\left(2 \mathrm{~L} 11^{\prime \prime} \times 1 / 8^{\prime \prime}\right)$ & 1.4291 & 411.58 & 37 & LC \\
F & $11\left(2 \mathrm{~L} 2^{\prime \prime} \times 1 / 8^{\prime \prime}\right)$ & 1.6655 & 479.66 & 27 & LC \\
G & $11\left(2 \mathrm{~L} 2^{\prime \prime} \times 1 / 8^{\prime \prime}\right)$ & 1.9034 & 548,18 & 37 & $\mathrm{UC}$ \\
\hline
\end{tabular}

Table 3. Results considering one to four groups

\begin{tabular}{cccccc}
\hline Configuration & $C_{1} *(\$)$ & $C_{2} *(\$)$ & $C_{3}^{*}(\$)$ & $C_{4} *(\$)$ & $C_{4} * / C_{1} *$ \\
\hline A & 490.86 & 460.05 & 429.29 & 387.39 & 0.789 \\
B & 490.86 & 430.01 & 399.28 & 369.16 & 0.752 \\
C & 490.86 & 430.01 & 399.28 & $\mathbf{3 6 3 . 2 5}$ & 0.740 \\
D & 490.86 & 460.05 & 429.29 & 387.38 & 0.789 \\
E & $\mathbf{4 1 1 . 5 8}$ & $\mathbf{4 1 1 . 5 8}$ & 411,58 & 381.48 & 0.927 \\
F & 479.66 & 421.60 & $\mathbf{3 9 0 . 8 7}$ & 382.26 & 0.797 \\
G & 548.18 & 473.07 & 408.79 & 372.79 & 0.680 \\
\hline
\end{tabular}


The values in bold in Table 3 indicate the configuration of lower cost in each analyzed situation. The last column shows the relation between the optimal cost of situations 4 and 1, for which an average reduction in total cost of $\sim 22 \%$ was observed. Among all the analyzed configurations, structure E presented the greatest regularity. It was observed that the internal vertical elements, although not stressed, were decisive for reducing the slenderness of the elements of the lower chord.

\subsection{Optimization of cross sections and geometry}

The presented study was complemented by allowing variation in the height of the upper chord of the trusses and by inclusion of their nodal coordinates in the set of design variables. The optimum heights obtained $\left(h^{*}\right)$ for situations 1 and 4 , as well as the new optimum cost and the consequent relative improvement, can be observed in Table 4, starting from the optimum values determined for the initial height of $1.00 \mathrm{~m}$. In this table, the subindex $m$ indicates the cost obtained for the new height, and $\alpha$ is the angle formed between the diagonals and the horizontal elements.

From Table 4, it is observed that the minimum costs are those corresponding to configuration $\mathrm{E}$ for both situations analyzed (with optimum values being in bold). It should be noted that, although the reductions in the optimal cost for the change in the ordinate of the chord have shown little significant in percentage terms, these reductions are in most cases due to small changes in this ordinate. For example, for configurations $\mathrm{A}$ and $\mathrm{D}, \mathrm{a}$ displacement of $4 \mathrm{~cm}$ upward in the top chord generates a reduction of $>11 \%$ in the total cost of the structure.

Fig. 4 shows the variation in the minimum weight of the truss relative to configuration $\mathrm{E}$ for a single group of elements as a function of the variation in the position of the upper chord (truss height). In the graph, the lines indicate the increase in total weight as the height also increases as a result of the growth in the length of the internal elements (diagonals and verticals). The discontinuities presented correspond to changes in the cross section of the elements. For trusses with height $<0.86 \mathrm{~m}$, there is a need to increase the section for the central elements of the lower chord, whereas, for heights slightly greater than $1.75 \mathrm{~m}$, the resistant internal forces of the extreme diagonals are exceeded.

From the results listed in Table 4, it can be observed that, in most cases, the optimum heights obtained were within the range suggested in the technical literature for the pre-dimensioning of the trusses, namely, a height between one fifth and one tenth of the span length [11] (in this case, between 0.90 and $1.80 \mathrm{~m}$ ). The angles of the diagonals in all situations analyzed lied outside the range suggested in the same references (between 40 and 45 degrees) as being the most economical.

Table 5 presents optimum costs and heights for configuration $\mathrm{E}$ obtained by varying the span between 9.00 and $45.00 \mathrm{~m}$. In all the structures generated, the distance between points of load application equal to $1.50 \mathrm{~m}$ was maintained, adding, therefore, between two subsequent analyses, a total of 6 nodes and 12 elements. The last column of this table indicates the relation between the optimal cost in which both four groups of elements and a single group were considered. A significant reduction was verified: from $14 \%$ for the shortest span to values $>42 \%$ for the highest span.

The weight of optimized trusses per linear meter is illustrated in Fig. 5, in which $P 1 m$ and $P 4 m$ were obtained by dividing the optimal weights of Table 5 by the corresponding span. These weights are compared to those obtained by section optimization of the same trusses composed of a single type of section but with a fixed height (equal to one tenth of the span), a situation designated in the figure as $P 1$. A significant increase in weight for the latter situation, compared to $P 4 m$, can be seen, ranging from $18 \%$ to $122 \%$.

\section{Conclusions}

This work presented an application of the simulated annealing method to determine the minimum weight in steel trusses composed of rolled profiles. Although the present work is specific to the optimization of trusses of parallel chords, it is emphasized that the applied procedure can be used 
Table 4. Results for the simultaneous optimization of cross sections and geometry

\begin{tabular}{ccccccccc}
\hline Configuration & $h_{1} *(\mathrm{~m})$ & $\alpha_{1} *\left({ }^{\circ}\right)$ & $C_{1 \mathrm{~m}} *(\$)$ & $C_{1 \mathrm{~m}} * / C_{1} *$ & $h_{4} *(\mathrm{~m})$ & $\alpha_{4} *\left({ }^{\circ}\right)$ & $C_{4 \mathrm{~m}} *(\$)$ & $C_{4 \mathrm{~m}} * / C_{4} *$ \\
\hline A & 1.04 & 34.73 & 433.87 & 0.884 & 1.04 & 34.73 & 375.12 & 0.968 \\
B & 1.70 & 48.58 & 447.64 & 0.912 & 0.92 & 31.52 & 363.14 & 0.984 \\
C & 1.70 & 48.58 & 447.64 & 0.912 & 0.92 & 31.52 & 357.72 & 0.985 \\
D & 1.04 & 34.73 & 433.87 & 0.884 & 1.04 & 34.73 & 375.12 & 0.968 \\
E & 0.86 & 48.91 & $\mathbf{3 8 8 . 0 5}$ & 0.943 & 0.86 & 48.91 & $\mathbf{3 3 3 . 3 9}$ & 0.874 \\
F & 1.17 & 57.34 & 443.29 & 0.924 & 0.92 & 50.81 & 348.88 & 0.913 \\
G & 0.60 & 38.66 & 526.18 & 0.960 & 1.03 & 53.94 & 347.96 & 0.933 \\
\hline
\end{tabular}

Table 5. Configuration E: optimum weight and height of variable spans

\begin{tabular}{cccccc}
\hline Span $(\mathrm{m})$ & $h_{1} *(\mathrm{~m})$ & $C_{1 \mathrm{~m}} *(\mathrm{kN})$ & $h_{4} *(\mathrm{~m})$ & $C_{4 \mathrm{~m}} *(\mathrm{kN})$ & $C_{4 \mathrm{~m}} * / C_{1 \mathrm{~m}} *$ \\
\hline 9.00 & 0.86 & 388.05 & 0.86 & 333.39 & 0.859 \\
12.00 & 1.24 & 698.71 & 1.09 & 590.37 & 0.845 \\
15.00 & 1.70 & $1,176.71$ & 1.29 & 949.85 & 0.807 \\
18.00 & 1.54 & $1,973.03$ & 1.22 & $1,435.48$ & 0.728 \\
21.00 & 1.57 & $2,956.32$ & 1.18 & $2,088.72$ & 0.711 \\
24.00 & 2.05 & $3,928.15$ & 1.75 & $2,959.54$ & 0.753 \\
27.00 & 2.59 & $5,152.06$ & 1.55 & $3,836.82$ & 0.745 \\
30.00 & 2.40 & $7,1864.06$ & 1.66 & $5,038.04$ & 0.701 \\
33.00 & 2.31 & $9,311.59$ & 1.87 & $6,183.53$ & 0.664 \\
36.00 & 2.75 & $11,436.77$ & 1.66 & $7,462.66$ & 0.653 \\
39.00 & 2.61 & $14,749.49$ & 1.71 & $8,876.65$ & 0.602 \\
42.00 & 3.03 & $17,651.12$ & 2.14 & $10,987.20$ & 0.622 \\
45.00 & 2.93 & $21,869.42$ & 2.04 & $12,597.61$ & 0.576 \\
\hline
\end{tabular}

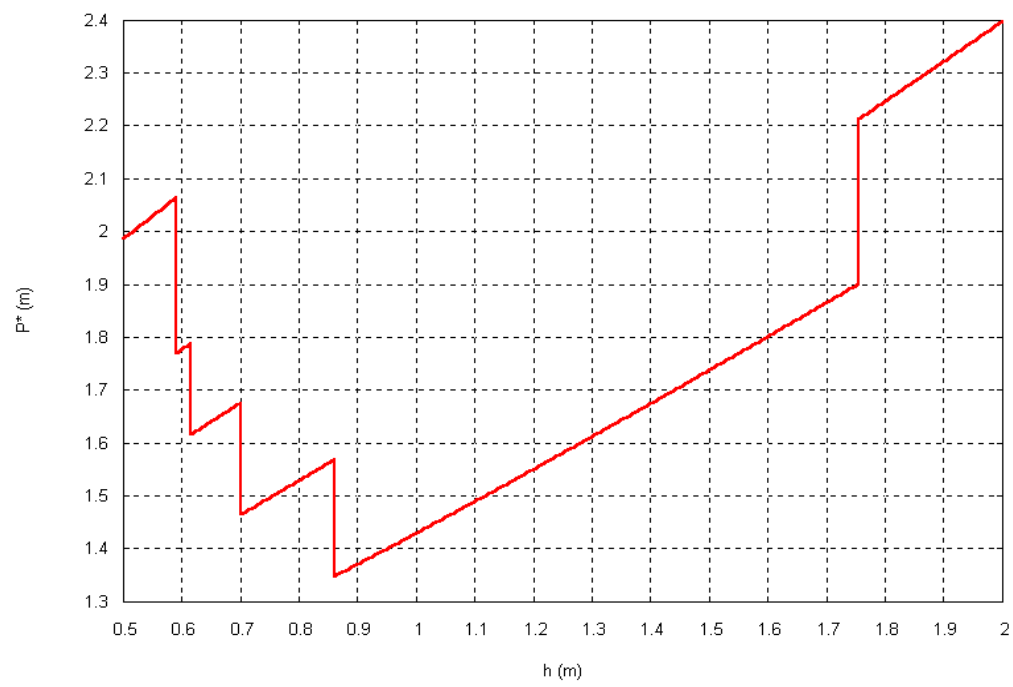

Fig. 4. Relation between total weight and height (upper chord position) 


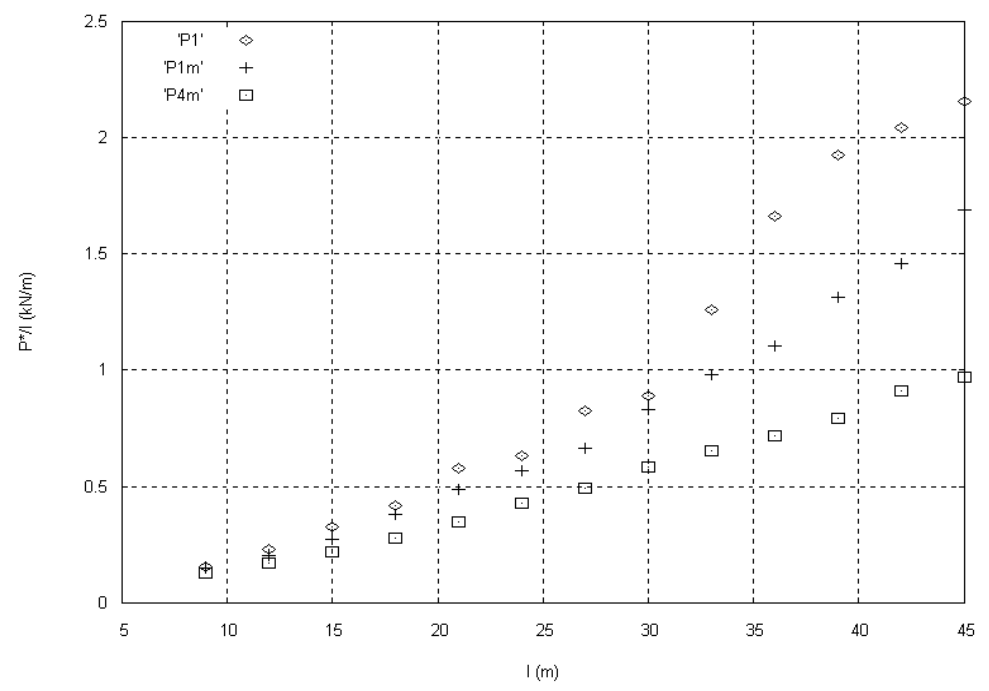

Fig. 5. Relation between optimum weight and span

for the analysis of several other configurations, owing to the generality of the formulation.

Regarding the examples presented, we observed great variation in the final weight of the structure as the number of groups of elements increases. By including truss height in the set of design variables, a significant additional cost savings was obtained. For the cases considered, the suggestion found in the technical literature for the relation between height and span was not valid, because it led to structures with higher cost than those obtained with optimization.

It must be stressed that, for the analyzed structures, the existence of constraints to the displacement out of the plane in all nodes was always considered, and the cost of the nodes has not been taken into account. Consequently, additional studies should be done to generalize the conclusions obtained in the present study.

The determination of the cross section and the geometry are predominantly performed according to the experience of the designer, and we have demonstrated that such optimization techniques can provide a useful auxiliary tool.

\section{Acknowledgments}

The first author thanks the Brazilian Council for Scientific and Technological Development-CNPq for the financial support granted.

\section{References}

[1] Sorensen K., Sevaux M., Glover F. A History of Metaheuristics. In ORBEL29-29th Belgian Conference on Operations Research (2015).

[2] Fister Jr. I., Mlakar U., Brest I., Fister I. A New Population-Based Nature-Inspired Algorithm Every Month: Is the Current Era Coming to The End? In StuCoSReC-3rd Student Computer Science Research Conference (2016).

[3] Kirkpatrick S., Gelatt C. D., Vecchi M. P. Optimization by Simulated Annealing. Science (1983) 671-680.

[4] Associação Brasileira de Normas Técnicas. NBR 8800 (NB 14): Projeto e Execução de Estruturas de Aço de Edifícios. Rio de Janeiro (1986) (in Portuguese).

[5] Stolpe M. Truss Optimization with Discrete Design Variables: A Critical Review. Structural and Multidisciplinary Optimization 5382) (2016) 349. 374.

[6] Michalewicz Z., Schoennauer M. Evolutionary Algorithms for Constrained Parameter Optimization Problems. Evolutionary Computation 4(1) (1996) 1-32. 
[7] Kripka M. Discrete Optimization of Trusses by Simulated Annealing. Journal of the Brazilian Society of Mechanical Sciences and Engineering 26(2) (2004) 01-04.

[8] Kripka M., Pravia Z. C. M., Medeiros G. F., Dias M. M. Simultaneous Geometry and Cross-Section Optimization of Aluminum Trusses. Multidiscipline Modeling in Materials and Structures 12 (2016) 315-325.

[9] Medeiros G. F., Kripka M. Structural Optimization and Proposition of Pre-Sizing Parameters for Beams in Reinforced Concrete Buildings. Computers and Concrete 11(3) (2013)1-18.

[10] Bordignon R., Kripka M. Optimum Design of Reinforced Concrete Columns Subjected to Uniaxial Flexural Compression. Computers and Concrete 9(5) (2012) 345-358.

[11] McCormac J. C. Análisis Estructural. México: Harla (1983) (in Spanish). 